\section{Impact of umbilical cord cleansing with $4 \%$ chlorhexidine on rate of omphalitis and separation time among newborns in Khartoum state, Sudan}

\author{
Abdelmoneim E.M. Kheir, ${ }^{1}$ \\ Amna M.A. Mustafa, ${ }^{2}$ Awatif A. Osman ${ }^{2}$ \\ ${ }^{1}$ Department of Paediatrics and Child \\ Health, Faculty of Medicine, University of \\ Khartoum; ${ }^{2}$ Nursing School, University of \\ Medical Sciences and Technology, \\ Khartoum, Sudan
}

\section{Abstract}

Infection of the umbilical cord remains high in developing countries with subsequent increase in neonatal mortality rates. This may be due to the practice of applying potentially harmful substances to the freshly cut cord. The aim of this study was to assess the impact of umbilical cord care with $4 \%$ chlorhexidine on the rate of omphalitis and separation time among newborns in Khartoum State. This was a quasi-experimental research design that was carried out in the main maternity hospitals of Khartoum state between February and August 2012. One hundred mothers and their respective babies were selected by convenience sampling and were divided equally into intervention and control groups. The tools used for data collection were a questionnaire and an observational checklist. The data were analyzed using Statistical Package for Social Sciences version 17 for descriptive and inferential statistics. Umbilical cord training was effective in enhancing mothers' knowledge and skills in the intervention group. The result showed that the omphalitis rate was reduced significantly in the intervention group; also the intervention group had a shorter separation time (mean=5.02) compared to the control group (mean=7.24). In conclusion, the application of $4 \%$ chlorhexidine on the freshly cut umbilical cord stump, significantly reduces omphalitis rate. This inexpensive and simple intervention can save a significant number of newborn lives in developing countries.

\section{Introduction}

Globally, there are approximately 3.3 million neonatal death each year, $98 \%$ of these occur in developing countries, more than half associated with home delivery, a third are due to infection. ${ }^{1,2}$

The umbilical cord stump constitutes a potential area for infection due to the presence of necrotic tissue that speeds up the colonization of organisms. About 2 to $7 \%$ of infants born in low resource countries develop omphalitis, $10 \%$ of these are severe with redness and discharge. Of all neonates with omphalitis, 2 to $15 \%$ die of a systemic infection or neonatal sepsis. ${ }^{3}$ While umbilical cord infections can occur in all settings, they are more likely to occur in developing countries, where the majority of births take place at home and are not attended by a skilled person. ${ }^{4}$

The high mortality rate among neonates in developing countries as a result of omphalitis can be attributed to the practice of applying potentially harmful substances such as animal dung to the cord stump after it is cut., ${ }^{5,6}$

Since 1998, the World Health Organization has recommended promotion of clean and dry cord care for newborn infants, while noting that topical antiseptics may be used where risk of infections is high. ${ }^{7}$ Topical antiseptic agents were used widely over the past years, despite a lack of conclusive evidence that these agents can reduce infection rates. ${ }^{8}$

In the past, chlorhexidine (CHX) was used widely in newborn intensive care units in western countries. ${ }^{3}$ Chlorhexidine is an antiseptic agent that reduces the risk of acquiring infections in different health care settings. It has a high safety profile with little bacterial resistance, it reduces infection rates as well as bacterial colony counts. ${ }^{9,10}$

Recently, three large community-based randomized trials have been conducted in Nepal, ${ }^{11}$ Pakistan $^{12}$ and Bangladesh ${ }^{13}$ to study the effectiveness of application of $4.0 \%$ CHX to the umbilical cord after birth; all 3 South Asian trials showed fairly similar, statistically significant protective effects against mortality with reduction rates ranging from $6 \%$ to $38 \%$.

The aim of this study is to i) assess the effect of umbilical cord cleansing with $4 \%$ CHX on rate of omphalitis and separation time among newborns in Khartoum state; ii) assess the existing knowledge scores of mothers towards umbilical cord care at pre-test; and iii) assess the existing skill scores of mothers towards umbilical cord care at post-test.

\section{Materials and Methods}

This was a quasi-experimental research design in which randomization is missing so it offers less tightness against bias. It was carried out in the main maternity hospitals of Khartoum state between February and August 2012. One hundred mothers and their respective babies were selected by convenience sam-
Correspondence: Abdelmoneim E.M. Kheir, Department of Paediatrics and Child Health, Faculty of Medicine, University of Khartoum and Soba University Hospital, P.0. Box 102, Khartoum, Sudan.

Tel.: +249.9.12313110 - Fax: +249.9 .183776295 .

E-mail: moneimkheir62@hotmail.com

Acknowledgements: the authors express their sincere appreciation to the administration of all the hospital in Khartoum for giving their approval and assistance in conducting this research. In addition, the authors are grateful to the mothers who participated willingly in this study. We dedicate this paper to Professor Awatif Ahmed Osman (co-author) who passed away recently.

Key words: Umbilical cord; Omphalitis; Chlorhexidine; Separation time; Newborn.

Conflict of interest: the authors declare no potential conflict of interest.

Contributions: the authors contributed equally.

Received for publication: 10 January 2015.

Revision received: 13 May 2015.

Accepted for publication: 13 May 2015.

This work is licensed under a Creative Commons Attribution 3.0 License (by-nc 3.0).

CCopyright A.E.M. Kheir et al., 2015 Licensee PAGEPress, Italy

Healthcare in Low-resource Settings 2015; 3:4946 doi:10.4081/hls.2015.4946

pling, which involves the selection of the most easily accessible members of the target population and were divided equally into intervention and control groups. In the intervention group the mothers were given health education about the importance of umbilical cord care and the risks of traditional practices on the umbilicus. The mothers were also told about the signs of omphalitis, on how to apply $4 \%$ CHX and to make sure that their hands were carefully washed before and after dressing. Mothers were told to apply CHX by a clean cotton wool daily for seven days, and then followed up at home three times on day 2,3 , and 7. The newborns were observed for occurrence of omphalitis, and the cord separation time. The signs of omphalitis included odor, erythema, swelling, and discharge. Omphalitis was graded into mild, moderate and severe depending on the number of signs present.

The inclusion criteria applied included full term babies on the first day of life without any complications at birth and who were either delivered by normal delivery or caesarian section. Preterm babies and those born outside hospital settings were excluded from the study.

The tools used for data collection were a questionnaire (Appendix) and an observation- 
al checklist. Immediately after birth, a pre-test questionnaire was filled for both the intervention and the control groups.

An immediate post training visit was carried out on day two to re-educate and observe the procedures, then on day three to check mothers' skills, signs of omphalitis and separation time. On day seven the third checklist and the post questionnaire were conducted and data collected. Home visits were extended up to 10 days for cord separation time.

The main dependent variables were knowledge score, skill score, omphalitis rate and separation time. The main independent variable was training on use of CHX, other independent variables were mother's age, education, parity, occupation, weight and gender of neonates.

The data were analyzed using Statistical Package for Social Sciences (SPSS) version 17 for descriptive and inferential statistics. Chi square test was used to study the correlation between the dependent and the independent variables, an independent sample t-test was used to examine the differences in knowledge scores between the intervention and the control groups. P value was set on an alpha level at 0.05 and $95 \%$ confidence limit.

Ethical clearance and approval for conducting this study was obtained from the ethical committee of the University Medical Sciences and Technology (IRB00008867) and permission from the hospitals was received before conducting the study. Prior informed consent was obtained from the mothers of the babies participating in this study after full explanation of the study.

\section{Results}

A total of 100 mothers and their respective babies were included in the study; they were divided equally into intervention and control group. Regarding mothers' education in the control group, 5 (10\%) were illiterate, 17 (34\%) received primary education, $14(28 \%)$ secondary education and 14 (28\%) were university graduates. Whereas in the intervention group, 8 (16\%) were illiterate, $15(30 \%)$ primary education, 13 (26\%) secondary education and $14(28 \%)$ were university graduates. In the control group 4 (8\%) mothers were employed and 46 (92\%) were unemployed, whereas in the intervention group 5 (10\%) mothers were employed and 45 (49.5\%) were unemployed. There were no significant differences between intervention and control group related to level of education, occupation, and parity at pre-test $(\mathrm{P}=0.75,0.5,0.59)$ respectively. Regarding mothers' age, the mean age in the control group was 26.32 (minimum 16 and maximum 43) and in the intervention group was 25.25 (minimum 16 and maximum 35).
Regarding baby's characteristics there were $26(52 \%)$ males and 24 (48\%) females in the control group, however there were 28 (56\%) males and 22 (44\%) females in the intervention group. The mean weight for babies in the control group was 3.035 (minimum 2.690 and maximum 4.500) whereas in the intervention the mean weight was 3.128 (minimum 2.500 and maximum 5.000). Concerning the gestational age in the control group, the mean was 38.48 (minimum 37 and maximum was 40 ) whereas in the intervention group the mean gestational age was 38.16 (minimum 37 and maximum 39). There were no significant differences between intervention and control group related to sex, weight or gestational age at pre-test. $(\mathrm{P}=0.6,0.44,0.5)$, respectively.

The study demonstrated that there were no significant differences of knowledge scores at pre-test between intervention and control group $(\mathrm{P}=0.412)$. The result supported the hypothesis that mothers in the intervention group and in the control group have equal knowledge at pretest. The 95\% confidence interval for the difference of Mean was ranging from - 1.978 to -0.818 (Figure 1).

The present study showed that there were significant differences of knowledge scores at post-test between intervention and control groups. The result was significant $(\mathrm{P}=0.001)$. The result supported the hypothesis that mothers in the intervention group have higher score compared to the control group. The 95\% confidence interval for the differences of mean was ranging from -11.10 to -9.06 (Figure 2).

The results showed that there were signifi-

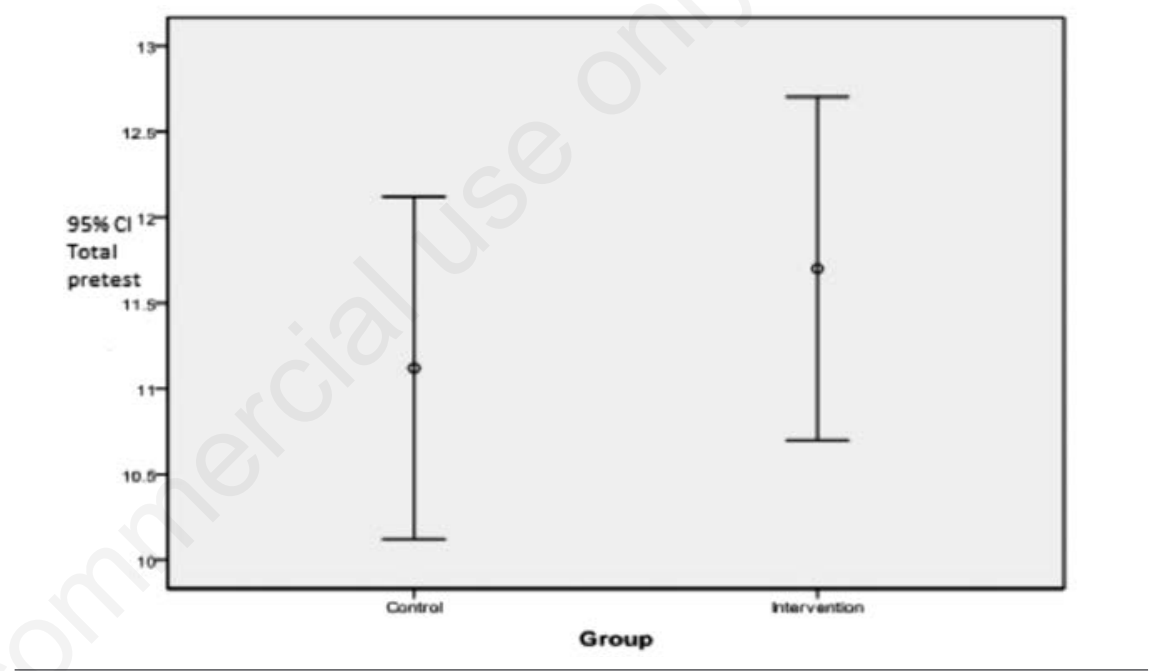

Figure 1. Error bars (two standard deviations above and below the mean) for the pre-test knowledge score for intervention and control groups.

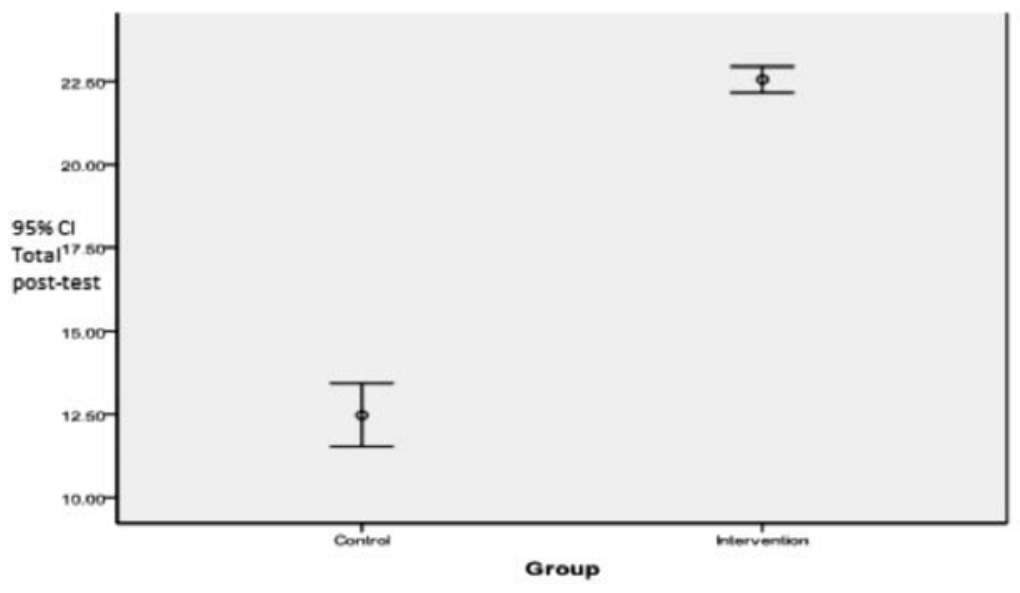

Figure 2. Error bars (two standard deviations above and below the mean) for the post-test knowledge score for intervention and control groups. 
cant differences of skills scores at post-test between intervention and control groups. The result was significant $(\mathrm{P}=0.001)$. The result supported the hypothesis that mothers in the intervention group have higher score compared to the control group. The $95 \%$ confidence interval for the differences of mean ranged from -3.09 to -2.59 (Figure 3).

A two-way contingency table analysis was conducted to evaluate whether the omphalitis rate in neonates who received umbilical cord care using CHX was lower compared to neonates without CHX (routine) umbilical cord care. The two variables were omphalitis and groups. Omphalitis with four categories (none, mild, moderate and severe), and the groups with two categories (intervention and control). The omphalitis rate in the control group was $64 \%$ calculated as: mild omphalitis $12 \%$, moderate $34 \%$, and severe $18 \%$. While in the intervention group there was no omphalitis with significant difference between the two groups ( $\mathrm{P}=0.001)$ (Figure 4).

An independent sample t-test was conducted to evaluate separation time between intervention and control groups. The result was significant $[t(98)=10.47, P=0.001]$. The result supported the hypothesis that neonates in the intervention group had shorter separation time (mean $=5.02$, Standard deviation $=0.74$ ) compared to the control group (mean $=7.24$, standard deviation $=1.30$ ). The $95 \%$ confidence interval for the difference of mean was ranging from 1.80 to 2.64 (Figure 5).

\section{Discussion}

Neonatal sepsis remains a major cause of neonatal mortality in the first two weeks of life. ${ }^{14}$ Around the globe and depending on the cultural background people apply harmful substances to the freshly cut cord stump, such as animal dung, ash or mud. This and other unhygienic exposures to the fresh wound could well account for a significant proportion of newborn sepsis. In the present study an attempt has been made to study the effect of umbilical cord cleansing with $4 \%$ CHX on rate of omphalitis and separation time among newborns in Khartoum state.

The study demonstrated that there were no statistically significant differences in demographic data between intervention and control groups. However, the knowledge score for both intervention and control group was low at pretest, which is different from a study done in Kenya where $40 \%$ of the mothers had good knowledge regarding care of the newborn umbilical cord. This difference could be attributed to increased level of education, living in middle class areas and increased maternal age..$^{15}$

Our study showed that there were signifi- cant differences of skills scores at post-test between intervention and control groups, which supported the hypothesis that mothers in the intervention group have higher score as

99.7\% of the mothers have good skills regarding hand washing and care of the umbilical cord. This is in contrast to a study done in rural Egypt where $43 \%$ of the mothers did not wash

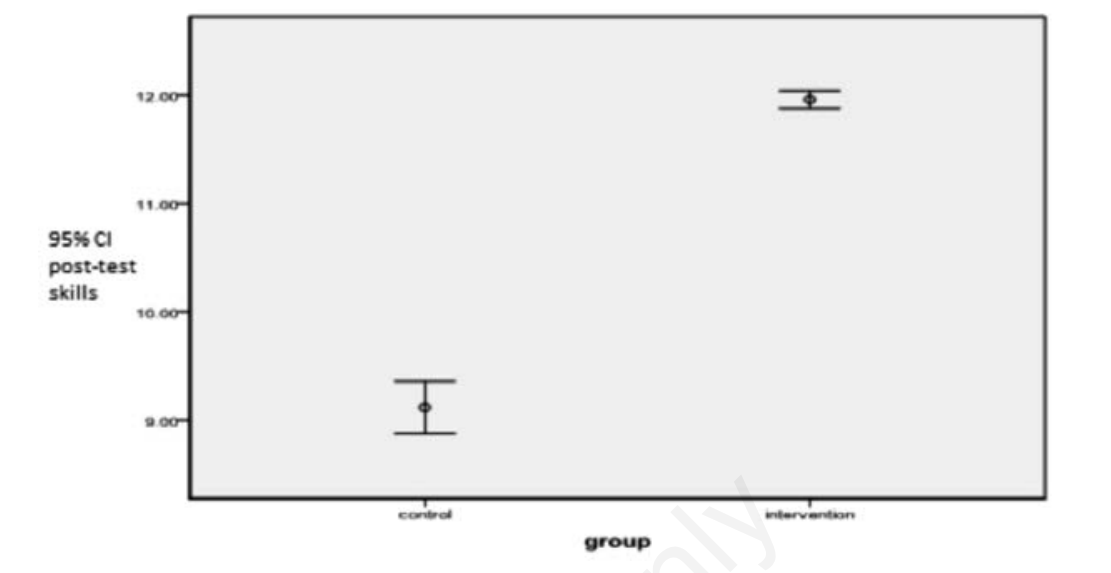

Figure 3. Error bars (two standard deviations above and below the mean) for the post-test skills score for intervention and control groups.

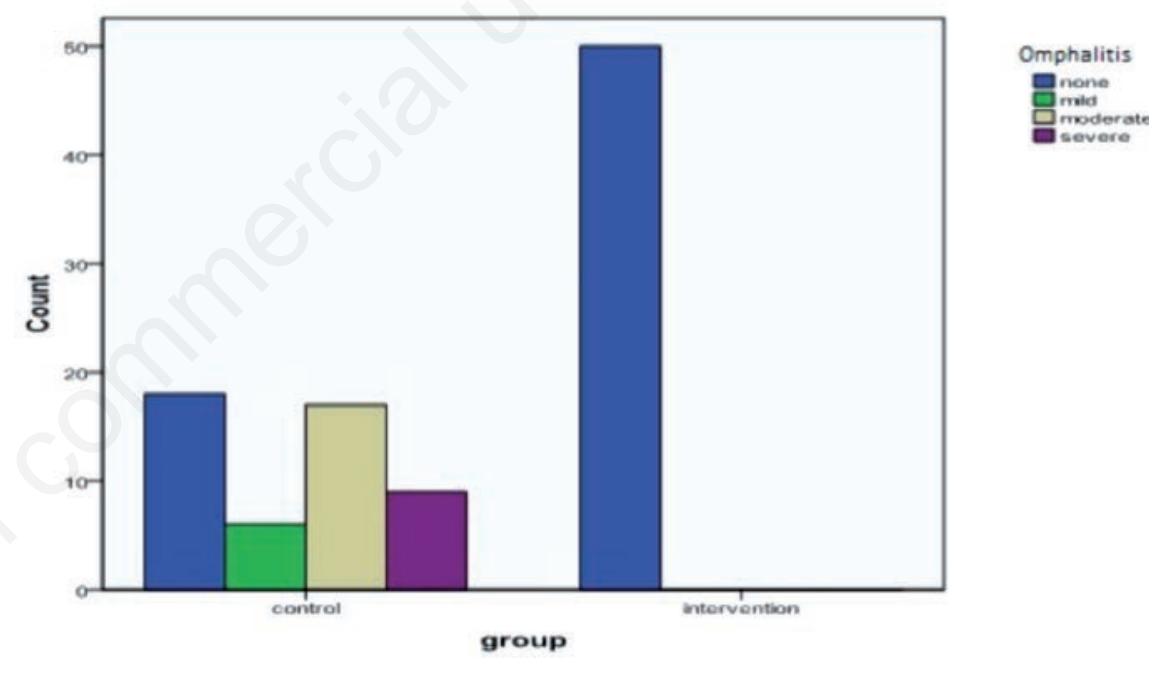

Figure 4. Cluster bar chart of omphalitis rate in the intervention and control groups.

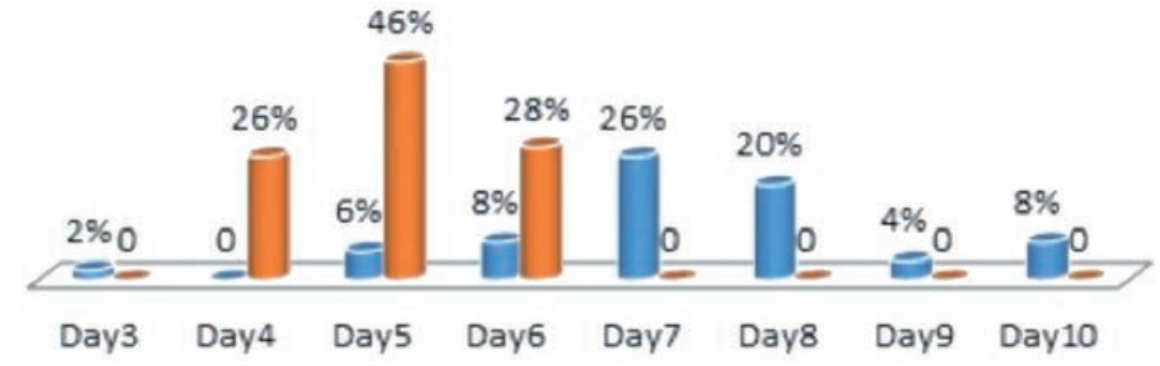

Figure 5. Cluster bar chart of cord separation time for intervention and control groups. 
their hands before neonatal care and only 7\% washed hands after diaper change. ${ }^{16}$ This difference is simply due to intervention as mothers in our study were well educated and visited many times at home.

The present study showed that the application of CHX to the umbilical cord of a newborn baby can significantly reduce the rate of omphalitis compared with routine care without CHX. The omphalitis rate in the control group was $64 \%$ compared with none in the intervention group. Similar results were obtained in a large cluster randomized study in Nepal involving over 15,000 infants delivered at home which compared the use of $4 \%$ CHX to the umbilical cord stump on 7 of the first 10 days of life with soap and water or dry cord care. They found a $75 \%$ reduction in severe omphalitis and a $24 \%$ reduction in neonatal mortality. Another study done in Pakistan involving 9741 infants, also delivered at home, used a factorial design to evaluate daily umbilical cord CHX treatment over 14 days and hand washing. They found no impact of hand washing on either the incidence of omphalitis or neonatal mortality, but a substantial reduction in both omphalitis and neonatal mortality in the CHX group. ${ }^{17}$

Our study demonstrated that neonates who received umbilical cord care using CHX had shorter separation time compared to neonates without CHX (routine) umbilical cord care (mean $=5.02$ ) compared to the control group (mean=7.24) with significant difference between the two groups. This is in contrast to many studies in this area which showed that topical CHX increased cord separation time by 25-50\%, which led to dissatisfaction among the caretakers who still accepted the intervention. ${ }^{18,19}$ This difference may be explained by the much larger sample size used in the other studies. However, further studies are definitely needed in this area.

\section{Conclusions}

Omphalitis is an important cause of neonatal mortality and preventing this condition and its associated neonatal mortality is of high public health importance. The use of $4 \%$ CHX on the freshly cut umbilical cord stump significantly reduces omphalitis rate. This inexpensive and simple intervention can save a significant number of newborn lives in developing countries.

\section{References}

1. Black RE, Cousens S, Johnson HL, et al. Child Health Epidemiology Reference Group of WHO and UNICEF. Global, regional, and national causes of child mortality in 2008: a systematic analysis. Lancet 2010;375:1969-87.

2. Oestergaard MZ, Inoue M, Yoshida S, et al. United Nations Inter-Agency Group for Child Mortality Estimation and the Child Health Epidemiology Reference Group. Neonatal mortality levels for 193 countries in 2009 with trends since 1990: a systematic analysis of progress, projections, and priorities. PLoS Med 2011;8:e1001080.

3. Mullany LC, Darmstadt GL, Tielsch JM. Role of antimicrobial applications to the umbilical cord in neonates to prevent bacterial colonization and infection: a review of the evidence. Pediatr Infect Dis J 2003;22:996-1002.

4. Mullany LC, Darmstadt GL, Katz J, et al. Risk of mortality subsequent to umbilical cord infection among newborns of southern Nepal: cord infection and mortality. Pediatr Infect Dis J 2009;28:17-20.

5. Agrawal PK, Agrawal S, Mullany LC, et al. Clean cord care practices and neonatal mortality: evidence from rural Uttar Pradesh, India. J Epidemiol Commun $\mathrm{H}$ 2012;66:755-8.

6. Darmstadt GL, Hussein MH, Winch PJ, et al. Practices of rural Egyptian birth attendants during the antenatal, intrapartum and early neonatal periods. J Health Popul Nutr 2008;26:36-45.

7. Capurro H. Topical umbilical cord care at birth: RHL practical aspects: RHL practical aspects. Geneva, Switzerland: World Health Organization; 2004.

8. Zupan J, Garner P, Omari AAA. Topical umbilical cord care at birth. Cochrane Db Syst Rev 2004;3:CD001057.

9. Goldenberg RL, McClure EM, Saleem S, et al. The use of vaginally administered chlorhexidine during labor to improve pregnancy outcomes: a systematic review. Obstet Gynecol 2006;107:1139-46.

10. McClure EM, Goldenberg RL, Brandes N, et al. The use of chlorhexidine to reduce maternal and neonatal mortality and morbidity in low-resource settings. Int $\mathrm{J}$ Gynecol Obstet 2007;97:89-94.

11. Mullany LC, Darmstadt GL, Khatry SK, et al. Topical applications of chlorhexidine to the umbilical cord for prevention of omphalitis and neonatal mortality in southern Nepal: a community-based, cluster-randomised trial. Lancet 2006; 367:910-8.

12. Soofi S, Cousens S, Imdad A, et al. Topical application of chlorhexidine to neonatal umbilical cords for prevention of omphalitis and neonatal mortality in a rural district of Pakistan: a community-based, cluster-randomised trial. Lancet 2012; 379:1029-36.

13. Arifeen SE, Mullany LC, Shah R, et al. The effect of cord cleansing with chlorhexidine on neonatal mortality in rural Bangladesh: a community-based, cluster-randomised trial. Lancet 2012;379:1022-8.

14. Liu L, Johnson HL, Cousens S, et al. Global, regional, and national causes of child mortality: an updated systematic analysis for 2010 with time trends since 2000 . Lancet 2012;379:2151-61.

15. Obimbo E, Musoke RN, Were F. Knowledge, attitudes and practices of mothers and knowledge of health workers regarding care of the newborn umbilical cord. E Afr Med J 1999;76:425-9.

16. Darmstadt GL, Hussein MH, Winch PJ, et al. Neonatal home care practices in rural Egypt during the first week of life. Trop Med Int Health 2007;12:783-97.

17. Goldenberg RL, McClure EM, Saleem S. A review of studies with chlorhexidine applied directly to the umbilical cord. Am J Perinat 2013;30:699-701.

18. Mullany LC, Shah R, El Arifeen S, et al. Chlorhexidine cleansing of the umbilical cord and separation time: a cluster-randomized trial. Pediatrics 2013;131:708-15.

19. Mullany LC, Darmstadt GL, Khatry SK, et al. Impact of umbilical cord cleansing with $4.0 \%$ chlorhexidine on time to cord separation among newborns in southern Nepal: a cluster-randomized, community-based trial. Pediatrics 2006;118:1864-71. 